\title{
Le cri et le bel canto. Konrad Klapheck, Gustave Moreau et les questions du sphinx
}

\author{
Scream and bel canto. \\ Konrad Klapheck, Gustave Moreau \\ and the questions of the Sphinx
}

\author{
EMMANUEL GUIGON \\ Musée des Beaux-Arts et d'Archéologie de Besançon (France)
}

Recibido : 4-XII-2015 Aceptado : 22-XII-2015

\section{RÉSUMÉ}

Ces pages servent de présentation à l'artiste allemand Konrad Klapheck en se proposant de centrer son approche sur les aspects les plus pertinents de sa fameuse série de " machines», particulièrement celui relatif à l'imaginaire, si proche de Lautréamont, des " machines à coudre». Klapheck, auquel André Breton dédie en 1965 le dernier des écrits publiés dans Le surréalime et la peinture, a élaboré depuis les années soixante une oeuvre de difficile adscription stylistique. En références aux propres déclarations réalisées par l'artiste dans La machine et moi (1997), ses recherches abordent les relations du motif du Sphinx et ses explorations par Gustave Moreau, et, le composant d'étrangeté cultivé dans la reproduction d'objets quotidiens à partir d'un fort sentiment d'identification avec les machines.

MOTS CLÉS

SPHINX, MACHINE À COUDRE, OBJETS QUOTIDIENS, ÉTRANGETÉ, DANGER

(C) Contrastes. Revista Internacional de Filosofia, vol. XXI-N³ (2016), pp. 177-191. ISSN: 1136-4076

Departamento de Filosofía, Universidad de Málaga, Facultad de Filosofía y Letras Campus de Teatinos, E-29071 Málaga (España) 


\section{ABSTRACT}

Theses pages serve as a presentation of the German artist Konrad Klapheck, focusing on highlighting the most important aspects of his well-known series of «machines», particularly focusing on the imaginary,so close to Lautréamont, of «sewing machines». Klapheck, to whom André Breton dedicated in 1965 the last of the writings contained in Le surréalisme et la peinture, has developed since the sixties a work of difficult stylistic allegiance. In reference to the statements made by the artist in La machine et moi (1997), its relations with the motif of the Sphinx and explorations inquired by Gustave Moreau and the element of estrangeness cultivated in the reproduction of everyday objects from a strong sense of identification with the machines.

SPHINX, SEWING MACHINE, EVERYDAY OBJECTS, STRANGENESS, DANGER

«[...]mais quand il se fut agi de coudre,

il n'eut pas son égal. Personne n'ignore, en effet, que les marins ont une aptitude remarquable pour le métier de couturière.» Jules Verne, L'Île mystérieuse, 1874.

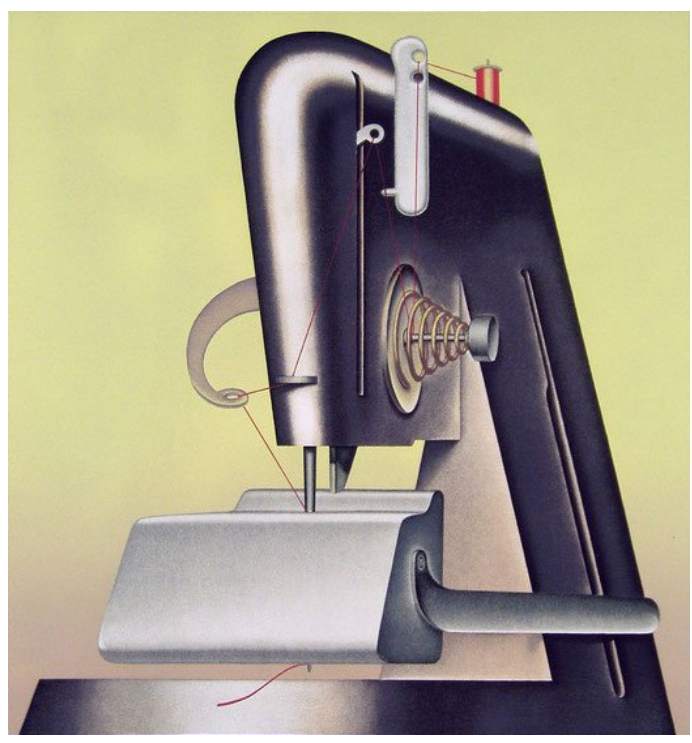

Fig. 1

LES QUESTIONS DU SPHINX (Die Fragen der Sphinx), tableau de 1984 [fig. 1] -longtemps exposé au musée de Grenoble- appartient à la série des « machines à coudre» que Konrad Klapheck peint depuis 1957. Comme dans tous ses tableaux, l'artiste utilise un style précis pour restituer une réalité objectale froidement décrite et puissamment stylisée. 
Les formes de la machine à coudre, minutieusement représentées, occupent toute la surface de la toile et lui confèrent ainsi une dimension monumentale. Un corps altier se dresse et s'incline, hiératique, sur un fond qui donne une impression de vide, en un mouvement qui s'organise autour d'un volant, de leviers et d'un fil rouge qui zigzague de la bobine au guide-filet à l'aiguille. Il y a dans la présence massive, solitaire et muette, de la machine à coudre, dans ses dimensions inhabituelles, dans son apparence à la fois conforme et simplifiée, de quoi dérouter légèrement, suggérant son éventuelle autonomie par rapport au monde des objets. Car cette image, aussi ressemblante qu'elle soit, interpose quelques interrogations entre l'esprit et l'objet qu'elle offre à la pensée. Elle est simple, sévère. Mais voilà. Elle n'est pas rassurante. Quand même elle représente un objet familier, elle contraint nos pensées à d'étranges détours. Elle nous piège. Elle nous traque. Elle nous menace. À une chose commune, instrument manufacturé, elle donne un air d'insolite que ne fait qu'accroître le titre. Comme dans tous ses tableaux, le titre semble destiné non pas à en faciliter la saisie, mais à la rendre plus complexe. Il introduit une tension, un supplément de sens, un éclairage nouveau, allusif ou évocateur d'une réalité, matérielle ou mentale, qui n'est pas figurée. «Le titre, précise Klapheck, ne doit pas être l'objet d'une recherche, il doit apparaître spontanément »(Klapheck 1997, 18).

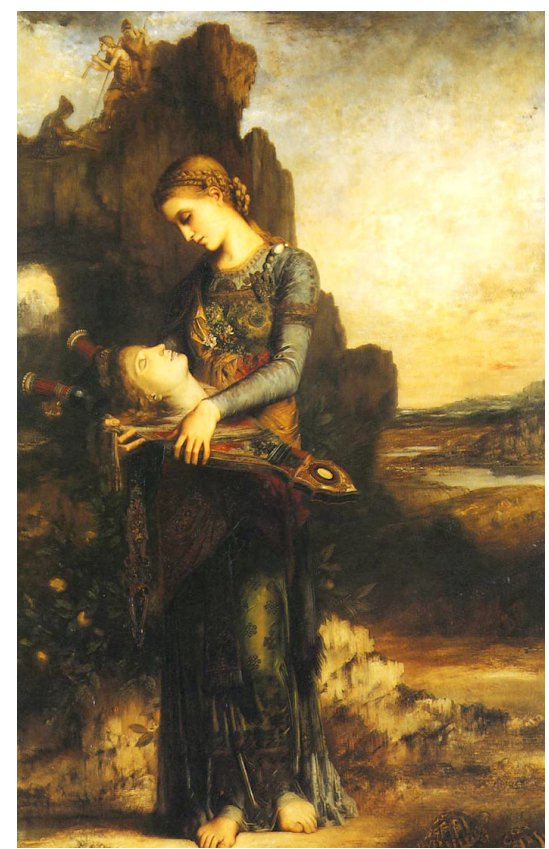

Fig. 2 
En arrivant à Thèbes, Edipe rencontra le sphinx. C'était un monstre à moitié homme et à moitié femme, qui posait des énigmes aux passants et dévorait ceux qui ne pouvaient lui répondre. Il demandait notamment : " Quel est l'être qui marche tantôt à deux pattes, tantôt à trois, tantôt à quatre, et qui contrairement à la loi générale, est le plus faible quand il a le plus de pattes ? » Il existait une autre énigme : «Ce sont deux sœurs, dont l'une engendre l'autre, et dont la seconde, à son tour, est engendrée par la première. » La réponse à la première devinette est « l'homme», parce qu'il marche à quatre pattes dans son enfance, puis sur ses deux jambes, et enfin s'appuie sur un bâton. La réponse à la seconde est « le Jour et la Nuit ». Edipe explique l'histoire et l'étonnement. Il dit le mot de l'énigme. L'œuvre de Klapheck pourrait faire référence au célèbre tableau d'Ingres Edipe explique l'énigme du Sphinx (1808). C'est une œuvre de sa jeunesse, conservée au musée du Louvre. À la demande de Serge Lemoine, Klapheck a choisi un tableau de Gustave Moreau, Orphée [fig. 2] de 1865 , pour participer à cette série de "Correspondances » organisée en 2008 par le Musée d'Orsay. La rencontre s'impose pour bien des raisons. Dans nombre de ses cuvres, Klapheck n'a cessé de dialoguer avec celles d'illustres prédécesseurs: Salvador Dali dans Séduction douce, en 1958 ; Fernand Léger dans La Chasse à la fortune, en 1978 ; Picasso à diverses reprises, comme dans Veuvage, en1959; Caspar David Friedrich et son tableau Les Phases de la vie pour Fiancées de soldats, en1967.

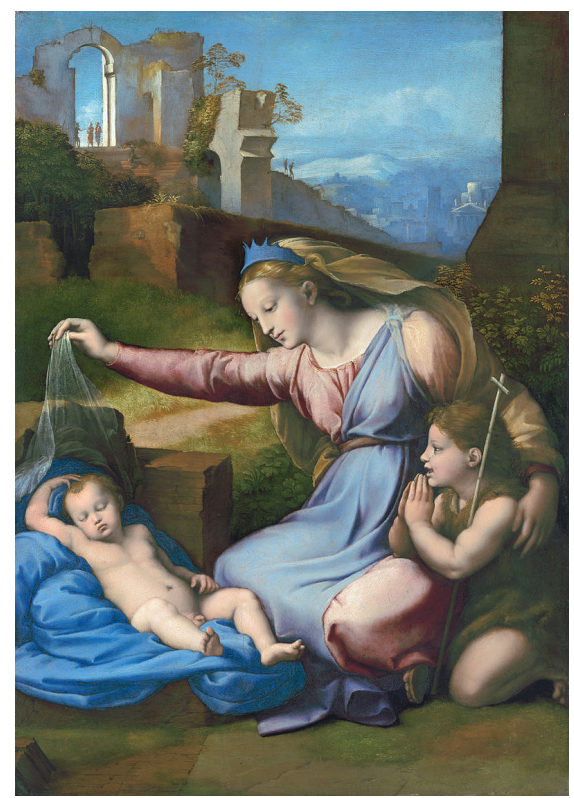

Fig. 3 
Mais ces références ne sont pas vraiment visibles. Elles interviennent au cours du travail. Elles n'en sont pas le point de départ.

C'est ainsi qu'il se remémora La Vierge au diadème bleu [fig. 3] de Giovanni Francesco Penni (vers 1512), anciennement attribuée à Raphaël, pendant l'élaboration de La Supermère [fig. 4], un tableau important dans son œuvre auquel il a apporté nombreuses transformations durant plus de vingt ans (Klapheck 1997, 45-51). Les allusions sont parfois ironiques.

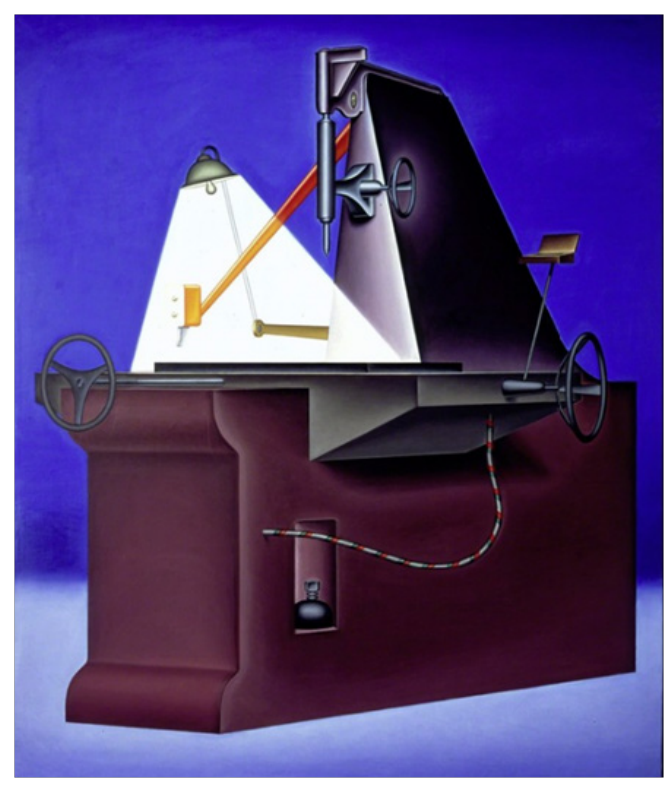

Fig. 4

Une photographie de Picasso dans sa baignoire lui inspire une série de robinets et de tuyaux de douche. Lors d'une visite au Prado à Madrid, ce n'est pas Vélasquez ou Goya qui lui suggère L'Homme adultère mais les extincteurs du musée. Pour Gustave Moreau, on connaît l'intensité avec laquelle il représente la figure du sphinx dans son œuvre. Dans son tableau Edipe et le Sphinx (1864), conservé au Metropolitan Museum of Art [fig. 5], l'interprétation qu'il fit du mythe grec est très influencée par l'œuvre d'Ingres exposée au Salon de1846 et de 1855 . Les deux artistes représentent le moment où CEdipe affronte le monstre ailé dans un passage de pierres à l'extérieur de la ville de Thèbes. La peinture de Moreau rencontrera un grand succès au Salon de 1864 et contribuera à la réputation de l'artiste, qui réalisa plus de trente études pour ce tableau, qu'il reproduira à plusieurs reprises. On a souvent insisté sur l'unité du «type physique» des héroïnes de Gustave Moreau : «Elles présentent presque 
toutes les mêmes caractéristiques, et toutes sont les sœurs du Sphinx », écrit Ragnar von Holten (1960, 7 y 12), qui rapproche par ailleurs ces figurations d'un poème de Heinrich Heine, dont le peintre possédait la traduction dans sa bibliothèque : «Devant la porte était un sphinx d'un aspect à la fois effrayant et attrayant, avec le corps et les griffes d'un lion, la tête et les seins d'une femme. La figure de marbre devint vivante. La pierre commençait à jeter des soupirs. Elle but toutes les flammes de mon baiser avec une soif dévorante. Elle aspira presque le dernier souffle de ma vie, et enfin, haletante de volupté, elle étreignit et déchira mon pauvre corps avec ses griffes de lion. »

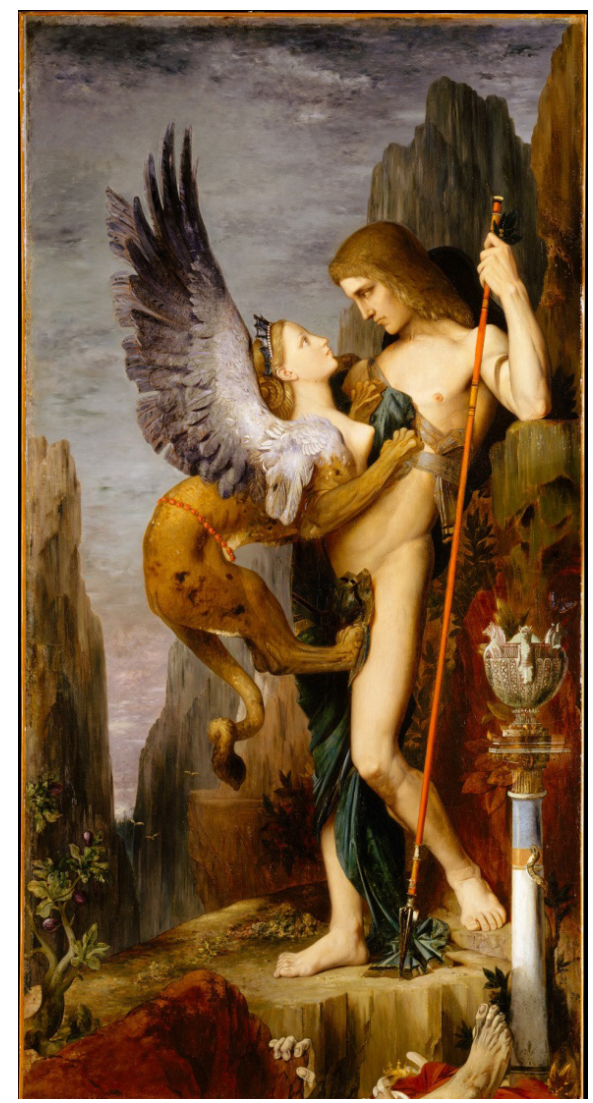

Fig. 5

On connaît le rôle joué par la découverte du Musée Gustave-Moreau dans la formation de la sensibilité d'André Breton-dont le dernier texte écrit sur la peinture, le 7 février 1965, est consacré à Konrad Klapheck. Le visitant dans 
son adolescence, Breton éprouva à la vue des célèbres figures féminines de Moreau (la Salomé, la Léda, La Fée au griffon...) un choc qui, dit-il, « a conditionné pour toujours ma façon d'aimer. La beauté, l'amour, c'est là que j'en ai eu la révélation à travers quelques visages, quelques poses de femmes. [...] J'ai toujours rêvé d'y entrer la nuit par effraction, avec une lanterne » (Breton 1965, 363). Les souvenirs des éloges décernés au peintre par Mallarmé et Huysmans achèvent d'expliquer que le peintre figure dès 1924 parmi les rares artistes du passé proches du surréalisme quand Breton le définit dans le premier Manifeste du surréalisme. Le 10 novembre 1926, Nadja Delcourt envoie à Breton une carte postale avec la reproduction d'Orphée de Gustave Moreau, tableau que le poète reproduira bien plus tard dans L'Art magique (1957) et représentant une jeune fille venant de recueillir la tête et la lyre. Au recto ce texte de Nadja : «Homme de pierre / Comprends-moi / Devant le mystère /Nadja $»^{1}$.

Dès ses débuts, à partir du milieu des années cinquante, Konrad Klapheck s'est choisi un registre qu'il n'a jamais quitté, toujours évident selon les œuvres, apparemment assez facile à définir, lié à la fois à une technique picturale et à un imaginaire, à ce qu'on peut appeler son matériel iconographique. Un dessin préparatoire, réalisé sur calque au moyen d'outils de mesure et de traçage semblables à ceux utilisés dans l'industrie est reporté sur la toile. La composition est construite sur des règles arithmétiques, comme la section d'or, selon un procédé qui s'apparente à celui de l'ingénieur ou de l'artisan méticuleux et donne tout son sens à la notion de «métier ». Le calque représente une phase préparatoire du travail, ce sur quoi l'artiste prend appui pour l'œuvre définitive, le support du dessin qui cherche forme, son questionnement, en quelque sorte le témoin du processus de création. Klapheck s'est donné des règles. Une méthode spécifique qui semble insensée en regard d'un travail de "représentation » et qu'il observe cependant depuis ses débuts avec une régularité obsessionnelle comme les commandements d'une religion personnelle. Ces règles sont librement acceptées et le pacte qui engage l'artiste est donc absolu. Elles renvoient la pensée vers une quête parfaitement originale, à laquelle il doit d'avoir accompli l'une des œuvres les plus singulières de ces cinquante dernières années. Klapheck en donne quelques exemples dans un texte de 1972, intitulé « Notes » : «En travaillant, je me laisse conduire par mon instinct, mais je parviens néanmoins à des règles intelligibles que, de nouveau, j'utilise systématiquement à l'exploration de mon inconscient. Par l'emploi rigoureux de la section d'or dans mes machines, je crée involontairement des monstres où je retrouve les désirs et les angoisses de mon enfance. » (Klapheck 1997, 17-18).

1 L'événement a fait l'objet d'une minutieuse analyse de Georges Sebbag dans son livre André Breton l'amour-folie, Suzanne Nadja Lise Simone, Paris, Jean-Michel Place, 2004, p. 95-96. 


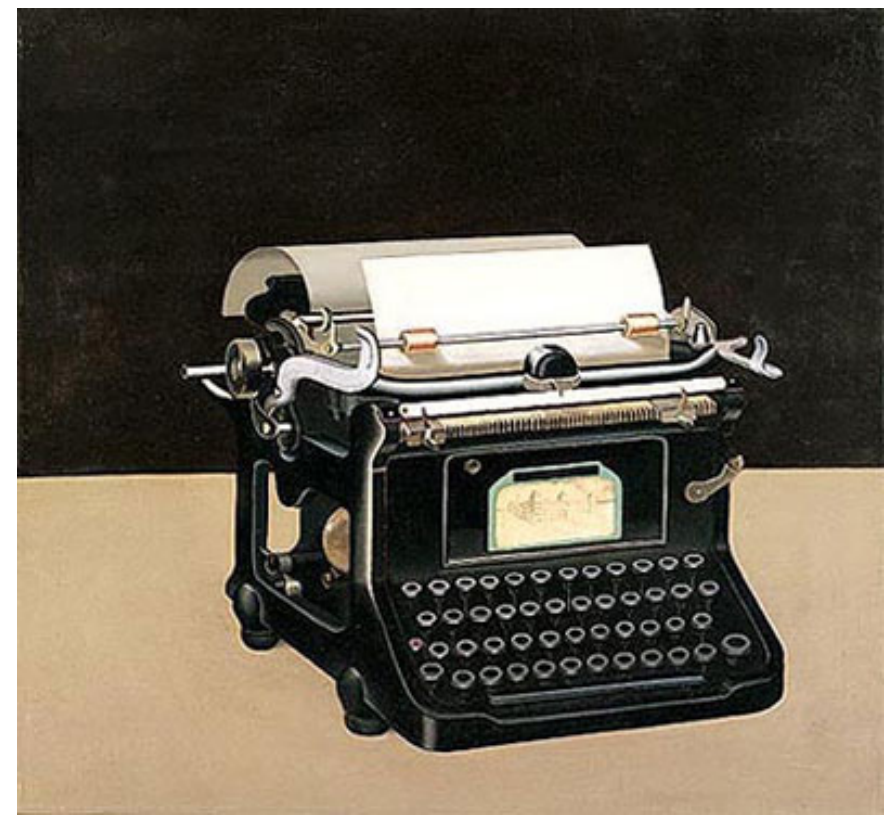

Fig 6

En 1955, à l'Académie des Beaux-Arts de Düsseldorf, le jeune peintre Konrad Klapheck -alors âgé de vingt ans- décide pour « se faire remarquer » de peindre « aussi précisément que possible » une vieille machine à écrire de la marque Continental. « Les trois semaines pendant lesquelles j’ai travaillé sur ma première machine à écrire comptent parmi les plus heureuses de ma vie. » Sa première œuvre, la seule portant un titre descriptif [ fig. 6], se plaçait à contre-courant du tachisme alors dominant. «Au vague je désirais substituer l'exactitude, à l'abstraction lyrique une réalité prosaïque exaltée » (Klapheck 1997, 13). C'est grâce à l'insistance amicale de Christian d'Orgeix que sera peinte la deuxième machine, deux ans après la première. Dans un texte de 1984, « Pourquoi je peins », l'artiste explique comment il a reconnu dans les lignes de la machine à coudre sa fiancée Lilo qu'il venait de quittera près une dispute. «Dans les lignes courbes du torse de la machine, dans la tête luisante avec guide-fil, pied et aiguille, je reconnus Lilo, de laquelle je m'étais séparé après une querelle. Une partie de mon chagrin et du sien était passée dans le tableau, que j'appelai pour moi seul d'abord je n'osais le dire à personne- $L a$ Fiancée mortifiée » (Klapheck 1997, 32-33) [fig. 7]. Klapheck a toujours peint par séries : machines à écrire, machines à coudre, téléphones et sirènes, timbres de bicyclette, robinets et douches, embauchoirs, fers à repasser, clés, cadenas, tuyaux de lance à incendie, couteaux suisses... La liste n'est pas limitative. 
«Chacune de ces catégories d'objets donne à la série de toiles correspondante un caractère différent $\gg$ (Klapheck 1997, 14).

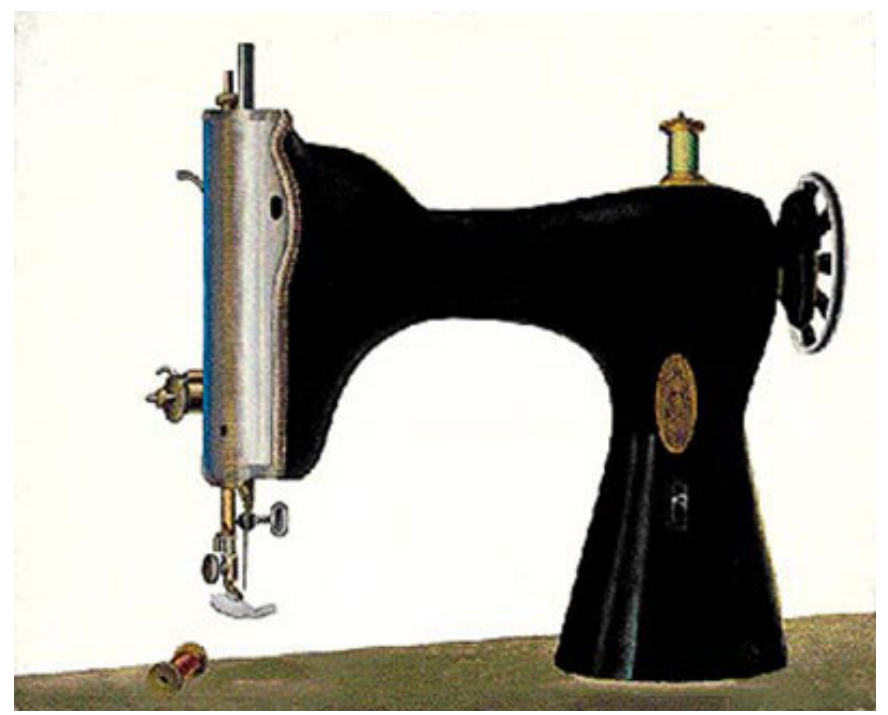

Fig. 7

Il s'agit toujours d'objets isolés de tout contexte et présentés en soi, reproduits avec minutie en des séries parallèles, ne laissant rien paraître des vibrations de la main. Ce sont des images au sens classique du terme: au premier abord réaliste, elles représentent toutes sortes de choses que chacun a vues ou pourrait voir et que pourtant on ne regarde pas en général. Elles sont empruntées à un modèle réel, à un imprimé publicitaire ou à une photographie, éventuellement prise par le peintre. Aucun doute possible, les objets représentés sont bien reconnaissables. Ils n'ont pas d'autre référent. Ils ne donnent pourtant pas la signification de l'œuvre. Ils ne sauraient renvoyer à aucune histoire déterminée qui aurait un développement et une fin. Ce sont en quelque sorte des objets en attente. Si le seuil de reconnaissance de l'objet fonctionnel est en effet toujours rationnellement lié à l'usage et à la nomination, et s'il peut être restreint à sa seule définition, à l'opposé, l'objet des peintures de Klapheck entrave à dessein tout espoir de le saisir : réellement, on voit et on imagine, les deux ensembles, comme fit le peintre pendant qu'il peignait le tableau. Sa peinture raconte de bien des façons cette forme de mise en garde à la manière des panneaux placés devant les passages à niveau non gardés : «Un objet peut en cacher un autre. » Cette idée de dédoublement du danger vient en quelque sorte nous surprendre ou nous prendre en défaut. La découverte du danger est 
capitale et déterminera durablement son travail: « Mais la machine se vengea de ma farce exhibitionniste. Sans que je l'eusse voulu, elle devenait un monstre insolite, étranger et familier en même temps, un portrait peu flatteur de ma propre personne. J'avais fait une découverte : à l'aide de la machine, je pouvais extraire du moi des mondes inconnus. La machine m'obligeait à confesser mes vœux et désirs les plus cachés » (Klapheck 1997, 14)

Klapheck a tenu à mettre en avant l'origine inconsciente de l'image, ainsi que son refus d'utiliser a priori telle valeur symbolique qu'elle aurait. « Je n'utilise pas les choses en tant que symboles, mais je les peins aussi bien que possible et je me laisse surprendre par ce qu'elles auront à dire. Finalement ce seront les tableaux qui devraient être plus intelligents que leur créateur et surpasser ses intentions. » Cette mise en garde faite, il précise à propos de la signification de ses thèmes d'élection : « La machine à écrire, instrument sur lequel les décisions les plus importantes de notre vie sont reformulées, est chez moi de sexe mâle. Elle représente le père, la politique, l'artiste. La machine à coudre, qui nous aide à dissimuler notre nudité, est femelle. Elle apparaît comme vierge, mère ou veuve » (Klapheck 1997, 14-15). La prédilection pour certains objets est généralement due à leur pouvoir d'évocation ou bien d'association en vue de réunir des éléments du langage et de les ordonner en fonction d'une sorte de mémoire du vécu. Chaque objet est lié à son usage, à son utilité, et peut engendrer une fiction. Se dessine alors un univers tissé de significations spécifiques dans lequel des tiens secrets unissent les objets entre eux. Dans son texte intitulé « Mes objets » (1973), il insiste ainsi sur les relations fonctionnelles ou formelles entre les familles d'objets, et les glissements qui permettent de passer de l'une à l'autre. Après avoir précisé que les douze objets les plus importants qu'on trouve dans ses tableaux « ont un ordre fixe déterminé par leur fonction et par leur aspect», il les passe en revue pour faire ressortir le fil secret qui les relie : « La machine à écrire est au service de la communication dans le monde de l'ordre abstrait. Les machines à calculer et les caisses enregistreuses appartiennent à la même famille. La machine à coudre, qui produit des vêtements, sert le monde du corporel. La perceuse fonctionne de la même façon et se rattache également à cette famille. La machine à écrire a des affinités formelles avec son antipode la machine à coudre : la touche devient bobine, le ruban devient fil, la matrice devient aiguille. » Les machines offrent ainsi toutes les possibilités de métamorphose en raison de leur proximité formelle, de l'échange éventuel de leurs fonctions ou des résonances affectives qu'elles peuvent susciter.

L'œuvre de Klapheck est hantée sans cesse par ce problème de rapports entre deux ordres de sens qui sont conjugués et se renvoient l'un à l'autre. Toujours, il semble vouloir penser ensemble des énergies contradictoires. « Ma peinture se situe au carrefour de diverses contradictions. » Le vertige 
naît souvent du minuscule saisi en un lieu gigantesque. Et le trop petit est au moins aussi inquiétant que le trop grand. Le peintre aime à la fois le mesurable et la démesure, à la fois ce qui rapproche et ce qui éloigne. Il n'oppose pas le désir de clarté au goût de l'équivoque, la spontanéité à la construction, le calme et l'inquiétude. «En peinture, le doute est mon guide. Je rejette et je corrige jusqu'à ce qu'il n'y ait plus rien à changer. Ma force naît de ma faiblesse [...] » (Klapheck 1997, 18). De fait, Klapheck va préciser : « Dans le dessin, j’aspire à une extrême dureté pour pouvoir me permettre la plus grande suavité de la couleur. J'aimerais combiner le cri et le bel canto [...]. J'essaie de donner à mes tableaux une surface lisse pour qu'ils aient l'air de ne pas avoir été faits par la main de l'homme. C'est pour donner plus de durée à mes passions que je les recouvre d'une couche de glace » (Klapheck 1997, 17-18). Il croit en la possibilité de faire coexister des espaces incompatibles, des matières hétérogènes, des théories antinomiques, des formes hostiles les unes aux autres. «Dans mes tableaux, il y a toujours sexe et crime, des caresses et des coups de poing, toujours les deux. Il faut beaucoup de tendresse, vraiment faire l'amour avec la toile, mais j'introduis beaucoup d'éléments agressifs pour convaincre le spectateur, saisir son attention » (Jouffroy 1990). Klapheck peint des situations conflictuelles. On en vient à penser les opposés ensemble. " C'est la souffrance qui me fait commencer mon travail et c'est le bonheur qui me conduit à l'interrompre. Par cette continuité du désir à l'accomplissement du désir, naissent et se développent les tableaux qui effacent les vestiges de la douleur et de la joie » (Klapheck 1997, 18-19). Jeux du vrai et du faux, lorsque l'image confirme l'incertain, lorsque les incertitudes viennent bouleverser nos mécanismes d'association, quand ce qui se donne à voir est à la fois proche et lointain, familier et déroutant, sans être totalement aberrant : dans nombre de ses tableaux, Klapheck traite la réalité comme un souvenir d'où le secret ne peut jaillir. Sans doute parce que le travail de la mémoire s'y trouve déjà accompli, et que ce travail persuade que tout ce qu'on peut apprendre de vérités en art se perd ou s'oublie. « Se montrer en se cachant, écrivait Jean Frémon, se cacher en se montrant, traquer de la vie derrière l'idéal de perfection de l'art dont l'œil et la main chaque jour tenteront d'approcher un peu plus, telle est, pour reprendre le titre de Michel Leiris, chez qui se croisent également les influences de Proust et de Roussel, la Règle du jeu de Konrad Klapheck » (Frémon 1990, 8). Seul demeure toujours présent dans la pensée ce qui marque l'image sensible. Cette image-là est le secret de toutes les images.

« C'est le regard de l'objet qui est essentiel. Il y a toujours des yeux dans mes objets : des vis, des ouvertures, des touches ou des boutons. » Certains objets peints par Klapheck nous étonnent plus que d'autres, même s'ils ont tous le même air de famille. Ils « font monde », pourrait-on dire. Comme s'ils constituaient une sorte d'autoportrait de l'artiste. Klapheck le suggère dans 
les quelques textes qu'il a publiés épisodiquement, et dès ses premières expériences, quand il définit sa pratique, celle de donner lieu, par des objets, non pas à une sorte d'autobiographie en peinture, mais à une mise en forme de celle-ci. Chacun d'eux joue de la ressemblance et de la différence. Ils se renvoient les uns aux autres et trouvent à s'articuler dans une trame de relations plus ou moins ramifiées et plus ou moins complexes. Toujours énigmatiques. L'artiste cultive précisément cette ambiguïté, reconnaissant à ses figures une dualité fondamentale : à la fois familières et étranges. Mais c'est peut-être davantage parce que leur auteur a voulu les personnifier en leur conférant une existence propre, une histoire qui nous les rend attachantes. On se surprend à être attendris par le désœuvrement d'une machine à coudre ou la solitude d'un ventilateur. «Mes tableaux doivent être considérés comme un tout, une épopée, dont les acteurs principaux ne sont pas l'homme, mais ses objets quotidiens les plus importants. Peut-être ceux-ci sont-ils plus capables que les portraits de leurs inventeurs de représenter la comédie humaine d'aujourd'hui » (Klapheck 1997, 19). Travail poétique, très certainement, que ce pouvoir, celui du devenir-être de chaque chose. Il y a là aussi une sorte de sortilège. «Peintre de machines, je suis moi-même devenu une machine. La goutte d'huile dont cette machine a besoin s'appelle inspiration. Qui serait plus apte à la fournir que l'amour? » Plus direct encore : le titre qu'il a donné en 1958 à un tableau qui représentait une machine à écrire au chariot d'une longueur insolite : Autoportrait athlétique (1958) [fig. 8], tandis que l'inscription « Star», dans Autobiographie en 1983, s'affiche sur le réservoir à essence de la motocyclette.

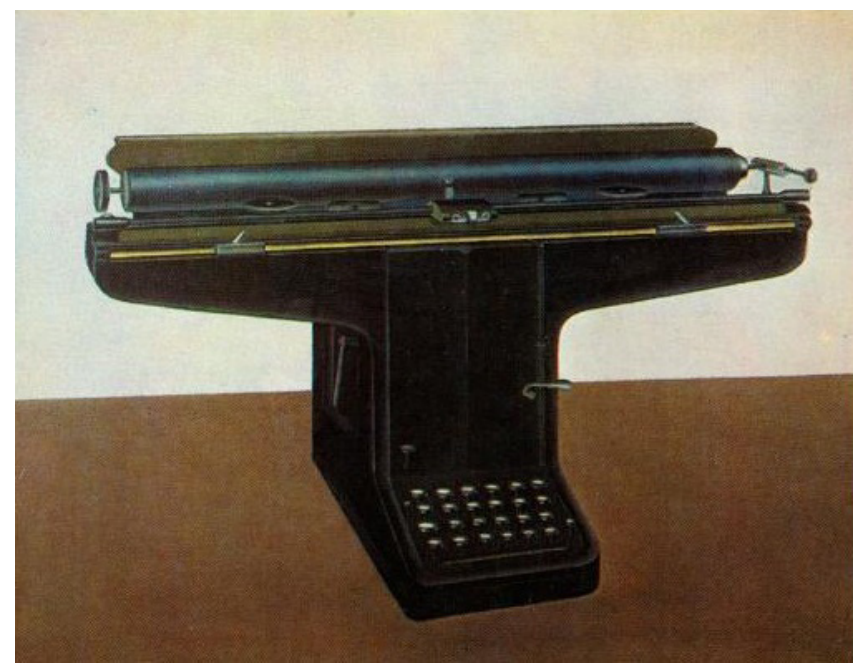

Fig. 8 
L'effrayant, c'est que l'art devienne parfois une sorte d'éphéméride dont l'artiste détache, chaque jour et chacune de ses années de vie, un ou plusieurs feuillets. Dérogeant aux représentations habituelles de l'artiste, Klapheck se situe intentionnellement à l'écart des modes, des grands éclats, des affirmations dogmatiques. Il cultive une extrême discrétion. Il aime aussi répéter « les clichés entendus mille fois ». C'est là « une façon de supporter la vie et les conventions, mais toujours en les ridiculisant. Je ne veux pas être un artiste maudit, ce n'est pas mon genre. Je suis toujours très fier si les gens me demandent: «Et vous, que faites-vous? Vous êtes avocat, ou médecin ?» Cela m'amuse » (Jouffroy 1990). Au reste, il y a dans son œuvre des intentions sans cesse renouvelées, des recherches insatisfaites, un jeu de variantes et de variations parfois infimes sur un thème réitéré, mais toujours réinventé. Cette recherche est à la fois timide et obstinée. Il s'agit pour lui de désapprendre les codes et les poncifs. Se lancer à chaque moment de nouveaux défis. Comme si chaque nouveau tableau devenait une prouesse, une nouvelle occasion de découvrir en lui-même des habitudes inconnues, des aptitudes à organiser autrement le champ pictural. La création d'art est une aventure qui excède, qui devant ce ou contredit la théorie. " À chaque nouvelle toile, il me faut oublier mes expériences comme mes théories et m'engager désarmé dans une lutte incertaine, afin que le résultat s'inscrive avec plus de certitude dans le programme de mes tableaux » (Klapheck 1997, 17). André Breton, dans le texte magistral qu'il a consacré à Konrad Klapheck en 1965, a observé avec pénétration comment l'analogie entre l'homme et la machine avait occupé, depuis Descartes, une place importante dans notre inconscient collectif. Breton écrit : " C'est au cœur même de cette forte analogie que Konrad Klapheck dispose une navette étincelante. Les instruments qu'il choisit de figurer sont choisis parmi nos plus proches auxiliaires mais le projet est de passer outre à leur usage spécifique, de manière à en imposer leur image magnifiée. L'obtention de ce résultat exige ici que la structure de cet objet soit rendue avec une rigueur et un dépouillement en quelque sorte théoriques, alors que ce ne sera pas trop pour les exalter, lui et l'espace qu'il s'accorde, de tous les feux du prisme. C'est de cette collision avec les arêtes vives alternant avec la caresse des belles courbures, par la grâce d'une lumière prenant sa source dans la vie intérieure, que Klapheck obtient de se hausser graduellement, par la voie des correspondances, à un autre palier où il a prise sur le psychologique et le sociologique, en fonction desquels, dans l'intitulation de ses œuvres, il est on ne peut mieux habilité à rendre compte de sa démarche. » Et Breton conclut: « Konrad Klapheck se tient dans la pose - et montre toutes les ressources- du charmeur : sur l'air de musique dont il a pouvoir de faire danser le serpent sous tout un lustre d'oiseaux chanteurs ». En 1997, Klapheck décide d'infléchir radicalement sa peinture en introduisant la figure humaine, jusqu'alors exclue. Il s'attache principalement à représenter des nus dans des intérieurs, inspirés de 
photographies érotiques anciennes. «Si la richesse du décor - commente Gérard Durozoi- remplace dans cette nouvelle série d'œuvres le vide qui entourait les machines, elle semble répondre à la volonté d'affaiblir la portée symbolique de la scène (toujours plus ou moins primitive, au sens freudien) représentée : il s'agit là de représentation, avec ce que le terme implique de théâtral, davantage que de simple figuration ou évocation » (Breton 1965, 411-412).

La métaphore a raison de dire que, dans l'acte de peindre, le désir du corps de l'autre vient en quelque façon se jouer. Et celle-ci est vraiment la métaphore originaire de l'art : celle qui assimile toute surface d'inscription de traces à la peau sensible d'un corps. En réponse à l'enquête d'une revue surréaliste sur les « représentations érotiques », en 1964, Klapheck dit la difficulté de s'exprimer sur ses « représentations érotiques en écrivant, parce que ce sont mes tableaux qui contiennent tout ce que je pourrais dire sur l'amour. Chez moi, peinture et amours ont en échange permanent. Si je regarde ma femme, je songe aux courbes sensuelles des machines à coudre que je désire dessiner et mes tableaux achevés me donnent des révélations sur l'amour » (Klapheck 1997, 52-53). Ces nouvelles peintures ne nous rassurent pas. Nous ne pouvons prétendre y lire des évidences. Elles renvoient à des situations souvent improbables où le vrai et le faux, le vraisemblable et l'incongru, échangent leurs propriétés en une vertigineuse et joyeuse jonglerie. Vous pénétrez, en frissonnant peut-être, dans un monde où les choses ne cessent de célébrer des noces toujours recommencées : dans la cuisine ou dans la salle de bains, sur le ring, derrière la fenêtre. Le résultat est déconcertant. Il nous renvoie à quelques-unes de nos hantises, quelque chose qui serait le portrait de chacun de nous. Il touche de la sorte à ce qui est une des fins propres de l'art. Si l'art est le lieu d'accueil de l'imaginaire, c'est qu'il est cette forme de pensée qui montre ce que tout réel a pour nous d'énigmatique. À l'image de la première machine à écrire dont les touches ne portent aucune lettre, ces images-là sont en souffrance de langage. Les questions du sphinx sont toujours pertinentes. Avant comme après la résolution de l'énigme, nous savons que nous savons peu sur l'humaine condition. Il y a bien une machine à coudre, avec son volant, ses leviers, son volant, son guide-fil, son aiguille, qui sont les éléments identifiables d'une certaine espèce de machine dite à coudre. L'effet repose sur la reconnaissance. Maison bute sur le mot. Les mots dont on se sert pourraient à la rigueur décrire en leurs moindres détails ces images ; au moins sont-ils utiles pour les identifier, pour les rappeler à la mémoire. Mais après ? Comment déceler l'étrangeté dont on éprouve le sentiment, alors que la facture des images et leurs références tendent à refuser le principe d'identité ? Vous dites : «Qu'est-ce qu'une machine à coudre ?» À coup sûr l'invention d'un certain Barthélemy Thimonnier. Mais d'abord est-il nécessaire de le savoir? La question ne se pose pas. Là n'est pas la question. Mieux vaut dire l'énigme de ce qui est. Non pas « quel nom donner », mais « comment donner un nom ». 
Comme la Sphinge au désert, Klapheck s'étonne. On n'attend nulle réponse. On s'étonne que cela soit ainsi. Les Grecs anciens croyaient qu'à s'approcher d'une réalité énigmatique l'esprit était menacé. Le fil rouge des Questions du Sphinx a bien été coupé une fois son travail accompli. «C'est ici, écrit Jacques Dupin -à propos d'une autre machine à coudre de Klapheck, Boîte de Pandore (1985)-, que l'aiguille plonge, traverse sa proie et conduit le fil. On ne peut dire combien c'est vif. Combien lent et précis. Pour un portrait divaguant. Un dévoilement de la mère dans sa relation avec le fil et le fils » (J. Dupin 1997, 8). Motus et bouche cousue. C'est en assumant le secret que Klapheck a défini depuis longtemps son espace singulier. « J'ai peint la machine pour créer l'exceptionnel, pour m'éterniser d'une manière sans pareille. Au lieu de cela, la machine m'a appris la vanité de l'existence et de ma propre personne. Devrais-je le lui reprocher? Je pense que non, parce que prendre connaissance de la vie, c'est savoir la supporter » (Klapheck 1997, 16).

\section{RÉFÉRENCES BIBLIOGRAPHIQUES}

KLAPHECK, K. 1997 : La Machine et moi. Paris : L'Échoppe.

RVON HOLten, R. 1960 : L'Art fantastique de Gustave Moreau. Paris : Jean-Jacques Pauvert.

BRETON, A. 1965 : Le Surréalisme et la Peinture. Paris : Gallimard.

FREMON, J. 1990 : «Une comédie humaine », Klapheck. Peintures et dessins. Paris :

Galerie Lelong, coll. « Repères » $\mathrm{n}^{\circ} 62$.

DUROzoI, G. 2005 : «Konrad Klapheck ; la découverte de soi par la peinture », Konrad Klapheck. Strasbourg : éditions des Musées de Strasbourg.

DUPIN, J. 1997 : "À l'âge de la violence ", Klapheck. Paris : Galerie Lelong, coll.«Repères», $\mathrm{n}^{\circ} 94$.

Jouffroy, A. 1990 : «Entretien avec Konrad Klapheck». Paris : Opus International, $\mathrm{n}^{\mathrm{o}} 118$.

Emmanuel Guigon es Director de los Museos de Besançon (Francia) 
